\title{
Two Kinds of Predictability in the Lorenz System
}

\author{
Peter C. Chu \\ Department of Oceanography, Naval Postgraduate School, Monterey, California
}

29 June 1998 and 6 October 1998

\begin{abstract}
The Lorenz system is used to discuss two kinds of predictability: the model sensitivity to inaccurate initial conditions (first kind) and to inaccurate boundary conditions (second kind). The first kind of predictability has been investigated for a long time, but not the second kind. It was found that the Lorenz system has a capability to detect both kinds of predictability since the boundary condition is represented by a model parameter, $r$. Two sensitivity runs are designed by perturbing the initial condition and the model parameter $r$ by the same small relative error $\left(10^{-4}\right)$, which is equivalent to $10 \%$ of the instrumentational accuracy for surface temperature measurement. Comparison of model output between the control run and the sensitivity runs shows that the model error growth and the growing period are comparable between the two kinds of predictability. This indicates the importance of preparing accurate boundary conditions in numerical prediction.
\end{abstract}

\section{Introduction}

Atmospheric and oceanic numerical models are usually initial-value and/or boundary-value problems. Change in either initial or boundary condition leads to a variation of model solutions. Much of the predictability research has been done on the response of model behavior to an initial value perturbation. Little effort has been made to study the response of model behavior to a boundary value perturbation.

Recently, Chu et al. (1998) found a large response of the National Center for Atmospheric Research (NCAR) Community Climate Model version 3 (CCM3) to a tiny Gaussian-type random sea surface temperature (SST) error. The model they used contains 18 levels in the vertical with a top at $2.917 \mathrm{mb}$ and uses spherical harmonics as horizontal basis functions with a trianugular truncation at wavenumber 21 (approximately a $5.6^{\circ} \times 5.6^{\circ}$ transform grid). The CCM3 was integrated from the 1 September climatology of the atmospheric and surface fields, which was provided by the NCAR Climate and Global Dynamics (CGD) Division. The surface boundary conditions were monthly sea and land surface temperatures (also obtained from NCAR CGD Division) linearly interpolated onto each time step $(20 \mathrm{~min})$. First, they integrated CCM3 for 16 months from 1 September to 31 December of the next

Corresponding author address: Prof. Peter C. Chu, Department of Oceanography, Naval Postgraduate School, Code OC/CU, Monterey, CA 93943-5000.

E-mail: chu@nps.navy.mil year (control run) and used the data between 1 January and 31 December of the second year for comparison. Second, they did an anomaly run. Three months into the control run, they added a tiny Gaussian-type random SST anomaly with zero mean and $0.05^{\circ} \mathrm{C}$ standard deviation generated by the FORTRAN random number generator to monthly SST data (first-year December to second-year December), then interpolated into each time step. The rest of the forcing was kept the same. The model was integrated from 1 December of the first year to 31 December of the second year. Third, they calculated the root-mean-square difference between the anomaly and the control runs over the global atmosphere and, surprisingly, found strong model responses, even in the monthly mean values. The global errors grow rapidly within the first 20 days: $0.028 \mathrm{~N} \mathrm{~m}^{-2}$ in the surface wind stress, $70 \mathrm{~W} \mathrm{~m}^{-2}$ in the net surface heat flux, and $5.7 \mathrm{~mm} \mathrm{day}{ }^{-1}$ in the net moisture flux.

Is this phenomenon (predictability regarding boundary condition error) universal? What is the basic physics involved? Does it deserve recognition as a second kind predictability problem? We will answer these questions in this note.

\section{Two-dimensional system}

We use the Cartesian coordinate system with $x, y$ as the horizontal coordinates and $z$ the vertical coordinate. The corresponding velocity components are $u, v$, and $w$. The temperature is represented by $T$. Let us take a two-dimensional (i.e., $v=0, \partial / \partial y=0$ ) convection problem depicted by Saltzman (1962) as an example 
to understand the physics of the boundary error propagation.

Consider a fluid to be of height $H$, with free upper and lower boundaries, between which a temperature contrast, $\Delta T=T(0)-T(H)$, is maintained externally. By virtue of the continuity equation a streamfunction, $\psi$, can be defined as follows:

$$
u=-\frac{\partial \psi}{\partial z}, \quad w=\frac{\partial \psi}{\partial x}
$$

The two-dimensional convection model consists of a horizontal vorticity equation,

$$
\frac{\partial}{\partial t} \nabla^{2} \psi=-\frac{\partial\left(\psi, \nabla^{2} \psi\right)}{\partial(x, z)}+\nu \nabla^{4} \psi+g \alpha \frac{\partial T}{\partial x},
$$

and a heat equation,

$$
\frac{\partial T}{\partial t}=-\frac{\partial(\psi, T)}{\partial(x, z)}+\kappa \nabla^{2} T
$$

where

$$
\nabla^{2} \equiv \frac{\partial^{2}}{\partial x^{2}}+\frac{\partial^{2}}{\partial z^{2}}, \quad \frac{\partial(f, g)}{\partial(x, z)} \equiv \frac{\partial f}{\partial x} \frac{\partial g}{\partial z}-\frac{\partial g}{\partial x} \frac{\partial f}{\partial z},
$$

and the constants $g, \alpha, \nu$, and $\kappa$ denote, respectively, the acceleration of gravity, the coefficient of thermal expansion, the kinematic viscosity, and the thermal conductivity. Such a system has lower boundary conditions

$$
\begin{aligned}
& T(x, 0, t)=T(0), \\
& \psi(x, 0, t)=\nabla^{2} \psi(x, 0, t)=0
\end{aligned}
$$

and upper boundary conditions

$$
\begin{aligned}
& T(x, H, t)=T(H), \\
& \psi(x, H, t)=\nabla^{2} \psi(x, H, t)=0 .
\end{aligned}
$$

Since $T(0)$ and $T(H)$ are observable or measurable quantities, it is quite possible for them to have errors. If we perturb the lower (or the upper) boundary with a small error,

$$
T(x, 0, t)=T(0)+\epsilon \Delta T,
$$

what is the model response to this boundary condition error? We have two choices to investigate this problem: (a) integrating the dynamic system (2) and (3) with and without the boundary condition error separately and comparing the difference and (b) transforming the boundary conditions into a forcing term. We will take the latter approach.

\section{Boundary errors transformed as forcing errors}

\section{a. Variable transform}

Let a new variable, $\theta$,

$$
\theta(x, z, t)=T(x, z, t)-\left[T(0)-\frac{T(0)-T(H)}{H} z\right],
$$

replace $T$; the horizontal vorticity and heat equations (2) and (3) become

$$
\begin{aligned}
\frac{\partial}{\partial t} \nabla^{2} \psi & =-\frac{\partial\left(\psi, \nabla^{2} \psi\right)}{\partial(x, z)}+\nu \nabla^{4} \psi+g \alpha \frac{\partial \theta}{\partial x}, \\
\frac{\partial \theta}{\partial t} & =-\frac{\partial(\psi, \theta)}{\partial(x, z)}+\frac{\Delta T}{H} \frac{\partial \psi}{\partial x}+\kappa \nabla^{2} \theta
\end{aligned}
$$

The upper and lower boundary conditions for $\theta$ are homogeneous:

$$
\theta(x, 0, t)=\theta(x, H, t)=0 .
$$

We notice that the boundary condition error [(6)] becomes an error in the forcing term, $(\Delta T / H)(\partial \psi / \partial x)$, in (9).

\section{b. Lorenz system}

Lorenz (1963) expanded the two dependent variables $\psi, \theta$ in double Fourier series in $x$ and $z$, and truncated the series to include a total of three terms:

$$
\begin{aligned}
a\left(1+a^{2}\right)^{-1} \kappa^{-1} \psi= & X \sqrt{2} \sin \left(\pi a H^{-1} x\right) \sin \left(\pi H^{-1} z\right), \\
\pi r \Delta T^{-1} \theta= & Y \sqrt{2} \cos \left(\pi a H^{-1} x\right) \sin \left(\pi H^{-1} z\right) \\
& -Z \sin \left(2 \pi H^{-1} z\right),
\end{aligned}
$$

where

$$
\begin{aligned}
r & =\frac{R_{a}}{R_{c}}, \quad R_{a}=g \alpha H^{3} \Delta T \nu^{-1} \kappa^{-1}, \\
R_{c} & =\pi^{4} a^{-2}\left(1+a^{2}\right)^{3} .
\end{aligned}
$$

Here $R_{\alpha}$ is called the Rayleigh number, and $R_{c}$ is a critical value. When $R_{\alpha}$ exceeds $R_{c}$, convection will be developed. The boundary error is transferred into the parameter error in $r$. Substitution of (11) and (12) into (8) and (9) leads to the Lorenz system (Lorenz 1963):

$$
\begin{aligned}
& \frac{d X}{d \tau}=-\sigma X+\sigma Y, \\
& \frac{d Y}{d \tau}=-X Z+r X-Y, \\
& \frac{d Z}{d \tau}=X Y-b Z .
\end{aligned}
$$

Here,

$$
\tau=\pi^{2} H^{-2}\left(1+a^{2}\right) \kappa t
$$

is the dimensionless time, while $\sigma=\kappa^{-1} \nu$ is the Prandtl number, and $b=4\left(1+a^{2}\right)^{-1}$ is the wavenumber parameter. The term $r X$ in Eq. (14) comes from the term $(\Delta T / H) \partial \psi / \partial x$ in Eq. (9) and thus a perturbation to the boundary condition $\Delta T$ is equivalent to a perturbation to the parameter $r$. We may call $r$ the boundary-forcing parameter. 


\section{c. Two kinds of predictability in the Lorenz system}

Usually we use the Lorenz system [(14)-(16)] to illustrate the predictability due to the initial conditions $X\left(\tau_{0}\right), Y\left(\tau_{0}\right)$, and $Z\left(\tau_{0}\right)$. We may call it the first kind predictability. The predictability due to the boundary condition error [(6)] is now represented by the parameter $r$ in the Lorenz system. We may call it the second kind predictability. The difference between the two is obvious. For the first kind predictability, the error is introduced only at the initial time instance $\left(\tau=\tau_{0}\right)$. However, for the second kind predictability, the error can be introduced at any time instance.

\section{Experiments}

We integrated the Lorenz system [(14)-(16)] under three different cases: the control run, the sensitivity to initial condition run, and the sensitivity to boundary condition run. The value $\Delta \tau$ is chosen as 0.01 for the dimensionless time increment. All three cases are integrated from $\tau=0$ to $\tau=50$.

\section{a. Control run}

We use the same initial conditions

$$
X^{(C)}(0)=0, \quad Y^{(C)}(0)=1, \quad Z^{(C)}(0)=0
$$

and the same values for model parameters

$$
r^{(C)}=28, \quad \sigma^{(C)}=10, \quad b^{(C)}=\frac{8}{3}
$$

as Lorenz (1963) for the control run.

\section{b. Sensitivity to initial error run}

We keep everything the same as the control run except the initial conditions

$$
\begin{aligned}
& X^{(I)}(0)=0, \quad Y^{(I)}(0)=Y^{(C)}(0)(1+\epsilon), \\
& Z^{(I)}(0)=0,
\end{aligned}
$$

where we introduce a small error $\epsilon$ in the initial condition. Here the superscript " $I$ " denotes the sensitivity to the initial error run.

\section{c. Sensitivity to boundary error run}

We keep everything the same as the control run except the parameter $r$,

$$
r^{(B)}=r^{(C)}(1+\epsilon),
$$

where we introduce a small error $\epsilon$ in the parameter $r$. Here the superscript " $B$ " denotes the sensitivity to the boundary error run.

\section{d. Selection of $\epsilon$}

Here we use the Lorenz system as a guide to investigate the atmospheric predictability. The accuracy of the surface temperature measurement is around $0.1^{\circ} \mathrm{C}$. If the temperature difference between the lower and upper boundaries $(\Delta T)$ is assumed to be $100^{\circ} \mathrm{C}$, the relative error in $\Delta T$ is around $10^{-3}$ under the current limitation of instrumentational accuracy. We take $10 \%$ of this value as the small perturbation, $\epsilon=10^{-4}$, to the initial condition and the parameter $r$.

\section{e. Numerical scheme}

We use the same numerical scheme as used by Lorenz (1963), the double-approximation procedure, to obtain the numerical solution. This scheme is much more accurate than the forward difference procedure.

\section{Model error growth}

The model error growth due to initial condition error, boundary condition error, or both can be obtained from the time evolution of the difference between the sensitivity runs and the control run. Thus, we use

$$
E^{(S)}(\tau)=\frac{\left\{\left[X^{(S)}(\tau)-X^{(C)}(\tau)\right]^{2}+\left[Y^{(S)}(\tau)-Y^{(C)}(\tau)\right]^{2}+\left[Z^{(S)}(\tau)-Z^{(C)}(\tau)\right]^{2}\right\}^{1 / 2}}{\left\{\left[X^{(C)}(\tau)\right]^{2}+\left[Y^{(C)}(\tau)\right]^{2}+\left[Z^{(C)}(\tau)\right]^{2}\right\}^{1 / 2}}
$$

to detect the model error growth. Here $S=I, B$.

The model error evolution shows a similar pattern for the two cases: a growing period and an oscillation period (Figs. 1, 2). During the growing period, the model error $E^{(S)}(\tau)$ increases from 0 at $\tau=0$ to an evident value $\left[E^{(S)}(\tau)>1\right]$ at certain value of $\tau$, which is called the error-growing period. During the oscillation period, $E^{(I)}(\tau)$ oscillates between 4.5 and 0.1 , and $E^{(B)}(\tau)$ oscillates between 5.0 and 0.2 . We may also notice that the error-growing period and magnitudes are comparable between $E^{(I)}(\tau)$ and $E^{(B)}(\tau)$. The dimensionless growing period is around 22 for both errors. 


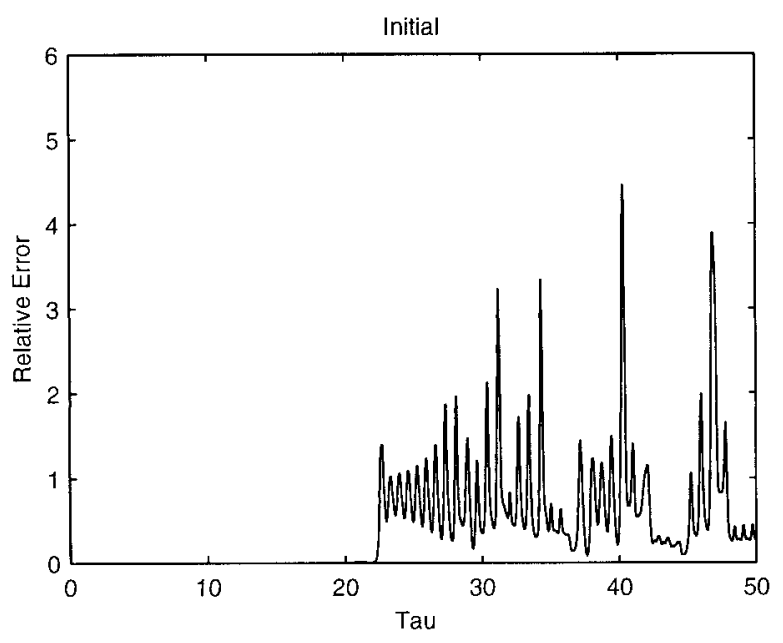

FIG. 1. Time evolution of the initial error $E^{(I)}(\tau)$.

\section{Dependence on parameter range}

We performed various numerical runs to show the dependence of model results on the three parameters: $r, \sigma$, and $b$. For each study, we vary only one parameter and then integrate the Lorenz system [(14)-(16)] for three different cases: the control run, the sensitivity to initial condition run, and the sensitivity to boundary condition run.

\section{a. Boundary forcing parameter $r$}

Keeping $\sigma$ and $b$ fixed as in (18), the boundary-forcing parameter $r$ is varied from 24 to 30. A summary of $E^{(I)}(\tau, r)$ and $E^{(B)}(\tau, r)$ after the model integration from $\tau=0$ to $\tau=50$ is presented in Fig. 3. The magnitude of $E^{(B)}(\tau, r)$ is basically comparable to the magnitude of $E^{(I)}(\tau, r)$ as $r$ varies. The maximum relative error exceeds 7 in both cases. The dimensionless growth period of $E^{(I)}(\tau, r)$ monotonically increases with the decreasing $r$ from 20 at $r=30$ to 50 at $r \approx 24$ (Fig. 3a). However, the dimensionless growth period of $E^{(B)}(\tau, r)$ has a minimum value of 20 at $r=28$ and increases as $r$ both increases and decreases. It reaches a value of 23 at $r=30$ and a value of 50 at $r \approx 24$ (Fig. 3b).

\section{b. Prandtl number $\sigma$}

Keeping $r$ and $b$ fixed as in (18), the Prandtl number $\sigma$ is varied from 5 to 15 . A summary of $E^{(I)}(\tau, \sigma)$ and $E^{(B)}(\tau, \sigma)$ after the model integration from $\tau=$ 0 to $\tau=50$ is presented in Fig. 4. The magnitude of $E^{(B)}(\tau, \sigma)$ is basically comparable to the magnitude of $E^{(I)}(\tau, \sigma)$ as $\sigma$ varies. The maximum relative error exceeds 7 in both cases. The dependence of dimensionless growth period on $\sigma$ is quite similar between the two cases. It has a minimum value of 17 at $\sigma=$ 15 and increases with decreasing $\sigma$ to a value of 50 as $\sigma$ approaches 5 .

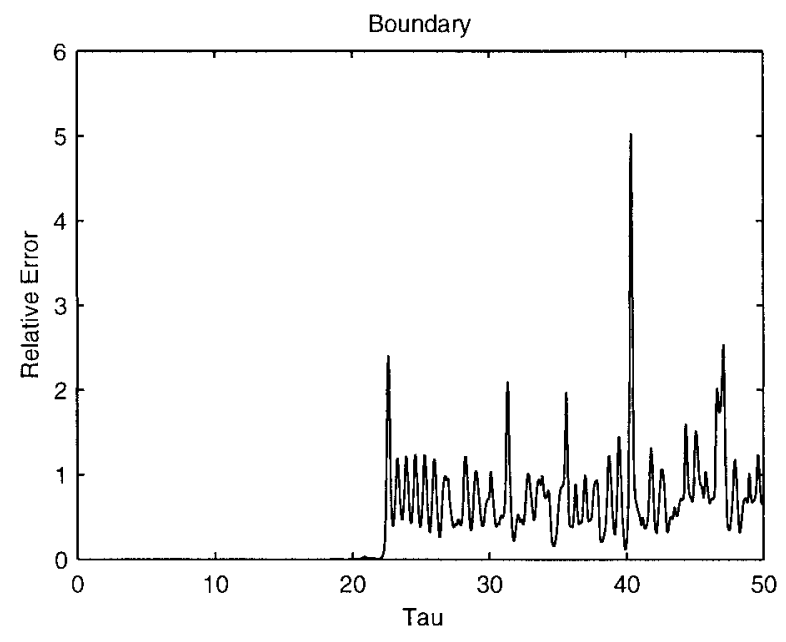

FIG. 2. Time evolution of the boundary error $E^{(B)}(\tau)$.

(a)

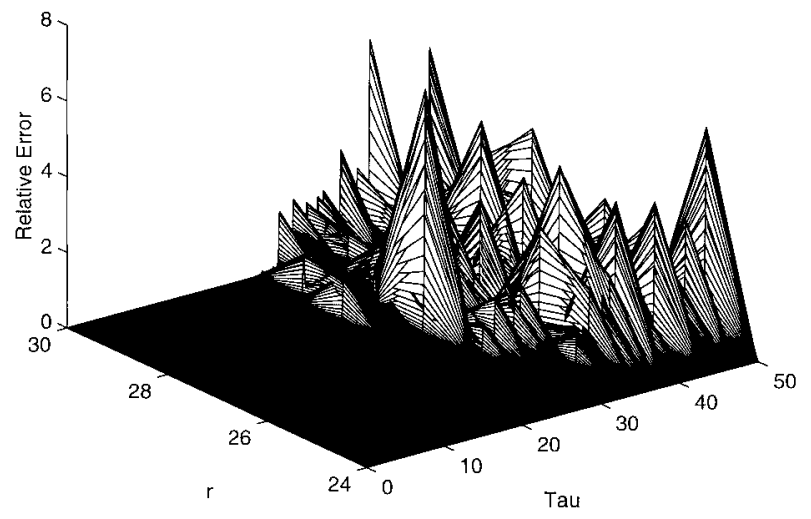

(b) Boundary

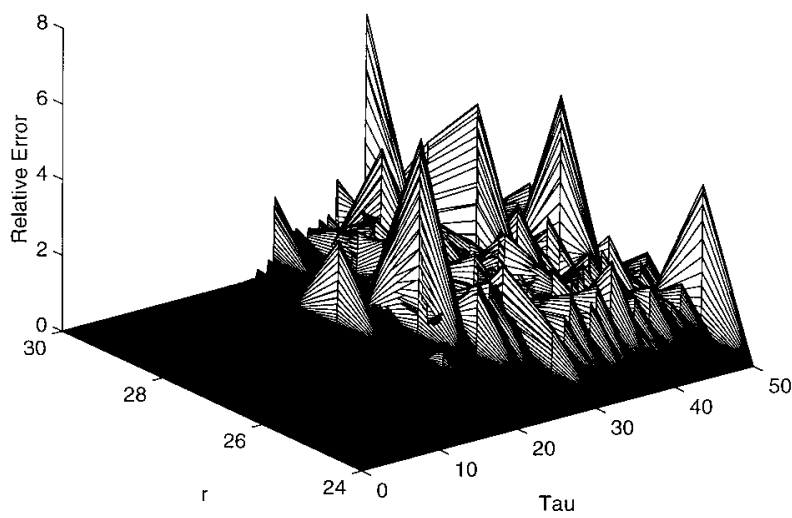

FIG. 3. Time evolution of relative error with varying boundary forcing parameter $r$ : (a) $E^{(I)}(\tau, r)$ and (b) $E^{(B)}(\tau, r)$. 
(a)

Initial

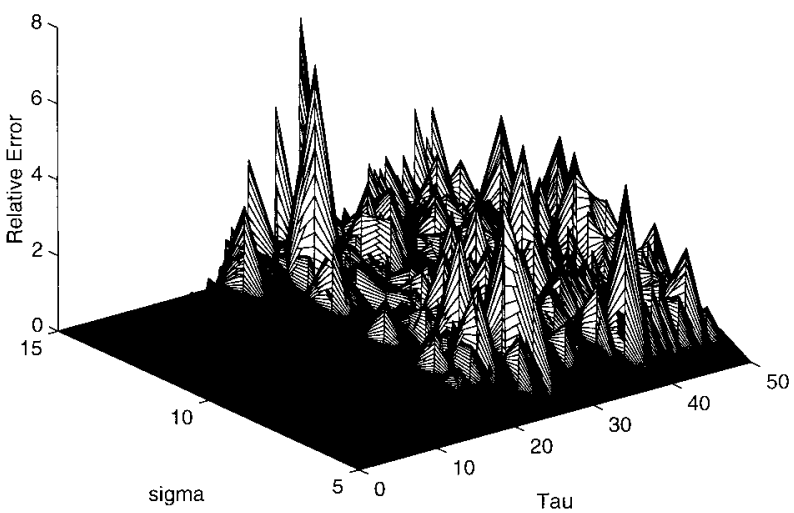

(b)

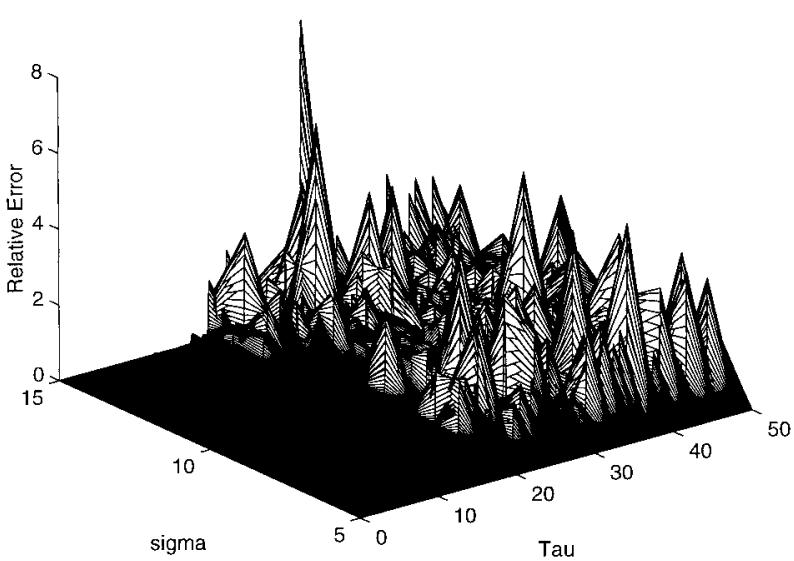

FIG. 4. Time evolution of relative error with varying Prandtl number $\sigma$ : (a) $E^{(I)}(\tau, \sigma)$ and (b) $E^{(B)}(\tau, \sigma)$

\section{c. Wavenumber parameter $b$}

Keeping $r$ and $\sigma$ fixed as in (18), the wavenumber parameter $b$ is varied from 2 to 4 . A summary of $E^{(I)}(\tau, b)$ and $E^{(B)}(\tau, b)$ after the model integration from $\tau=0$ to $\tau=50$ is presented in Fig. 5. The magnitude of $E^{(B)}(\tau, b)$ is basically comparable to the magnitude of $E^{(I)}(\tau, b)$ as $b$ varies. The maximum relative error exceeds 6 in both cases. The dependence of dimensionless growth period on $b$ is quite similar between the two cases.

\section{Conclusions}

1) Within the nonlinear dynamics community, there are three types of sensitivity that are commonly discussed: sensitivity to initial conditions, sensitivity to parameters, and sensitivity to boundary conditions. The first type of sensitivity has received a great deal of attention, the second has received a good deal of attention, and the third has received relatively little attention. Since the present study considers only a change in the boundary value $T(0)$, which is a parameter, we are studying only a very special case of sensitivity to boundary conditions, and this special case is equivalent to sensitivity to parameters.

2) Two kinds of predictability problems exist in the Lorenz system, namely, the model sensitivity to initial (first kind) and boundary (second kind) perturbations. The effect of the boundary error on the model can be represented as a forcing term.

$3)$ Introducing the same small relative error $\left(10^{-4}\right)$ to either the initial or boundary condition, the Lorenz system has a growing period and an oscillation period. During the growing period, the model error increases from 0 to an evident value larger than 1 . During the oscillation period, the model error oscillates between two evident values.

4) For the wide range of the parameter space, both the error-growing period and the relative error are com-

(a)

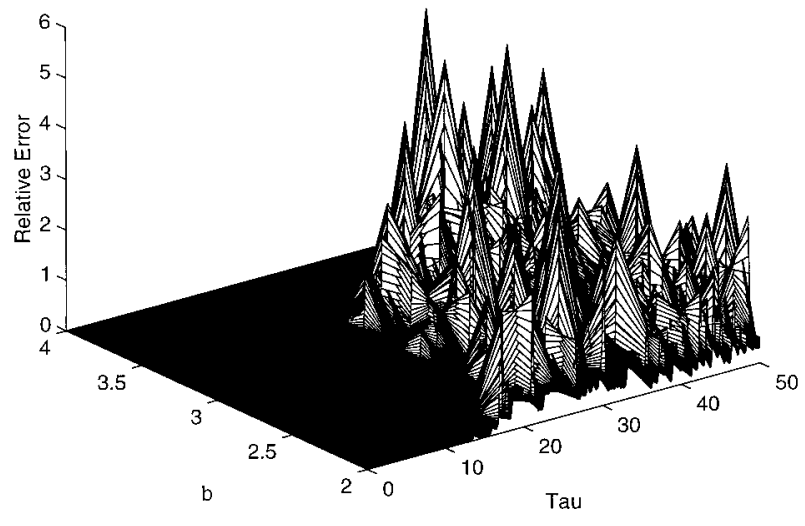

(b) Boundary

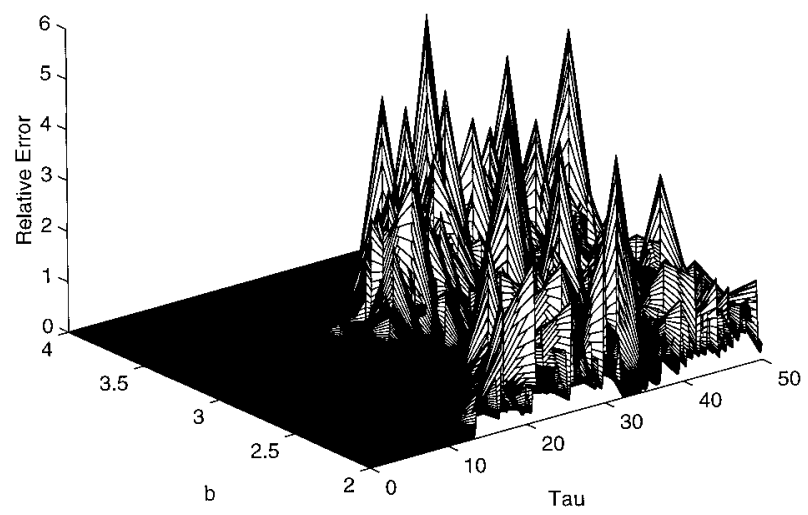

FIG. 5. Time evolution of relative error with varying wavenumber parameter $b$ : (a) $E^{(I)}(\tau, b)$ and (b) $E^{(B)}(\tau, b)$. 
parable between the first kind of problem (initial inaccuracy) and second kind of problem (boundary inaccuracy). This suggests the importance of preparing accurate boundary conditions for numerical prediction.

Acknowledgments. The author is grateful to Dr. Martin Claussen and two anonymous reviewers whose comments improved the manuscript a great deal. Many thanks to Chenwu Fan and Shihua Lu for programming assistance. This work was funded by the Naval Ocean- ographic Office, the Office of Naval Research Naval Ocean Modeling and Prediction Program, and the Naval Postgraduate School.

\section{REFERENCES}

Chu, P. C., S. H. Lu, and Y. C. Chen, 1998: Second kind predictability in climate models. Preprints, Ninth Symp. on Global Change Studies, Phoenix, AZ, Amer. Meteor. Soc., 86-90.

Lorenz, E. N., 1963: Deterministic nonperiodic flow. J. Atmos. Sci., 20, 130-141.

Saltzman, B., 1962: Finite amplitude free convection as an initial value problem-I. J. Atmos. Sci., 19, 329-341. 